\title{
RETRATAMIENTO DE UN CASO CON ATROFIA ÓSEA COMPLEJA COMBINADA UNIDA A PERIIMPLANTITIS. IMPORTANCIA DE LA REVERSIBILIDAD DE LOS TRATAMIENTOS IMPLANTOLÓGICOS
}

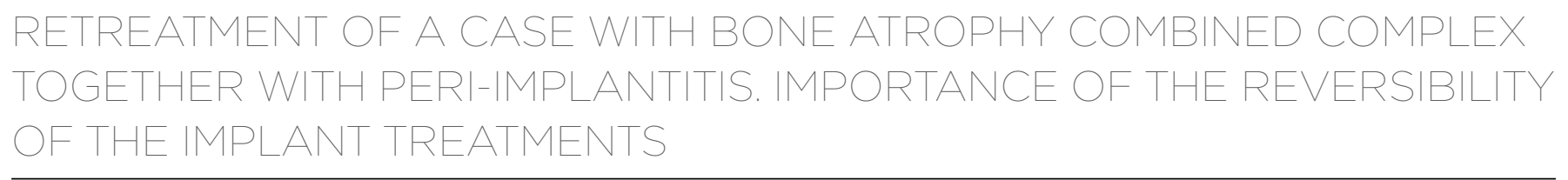

Eduardo Anitua' * (1)

eduardo@fundacioneduardoanitua.org

Artículo recibido: 26/05/2020

Arbitrado por pares

Artículo aceptado: 07/07/2020

Artículo publicado: 24/08/2020

* Autor corresponsal:

Eduardo Anitua

eduardo@fundacioneduardoanitua.org

\section{Resumen}

Los implantes dentales son un tipo más de tratamiento para la resolución de casos de edentulismo. Desde su inicio, en los años 70, han sido una alternativa creciente para rehabilitar pacientes, sobre todo en casos en los que las rehabilitaciones convencionales no eran posibles. Cada vez de forma más frecuente nos enfrentamos a retratamientos de implantes y casos con atrofias más complejas. En el siguiente caso, mostramos un retratamiento de implantes fracasados unido a una situación de atrofia extrema vertical y horizontal combinada. En casos de atrofia severa maxilar y mandibular, el uso de los implantes cortos puede acortar los tiempos de tratamiento y simplificar las técnicas quirúrgicas sin renunciar a la predictibilidad como en el caso mostrado.

Palabras clave: retratamiento, atrofia ósea, implante dental, periimplantitis

\begin{abstract}
Dental implants are an alternative treatment for extended the resolution of cases of edentulism. Since its inception in the year 70 have been a growing alternative to rehabilitate patients, especially in cases where conventional rehabilitations were not possible. Every time, in the dental office were more often we re-treatments of implants and cases with more complex atrophies. In the following case we show a re-treatment of failed implants attached to a situation of extreme atrophy combined vertical and horizontal. In cases of severe maxillary and mandibular atrophy, the use of short implants can shorten treatment times and simplify surgical techniques without sacrificing predictability as in the case shown.
\end{abstract}

Keywords: Re-treatment, bone atrophy, dental implant, peri-implantitis

\section{Introducción}

La implantología dental nos aporta soluciones para poder abordar diversos casos de edentulismo desde su comienzo en la década de los 70, hasta hoy, siendo una opción terapéutica adecuada para aquellos casos donde los tratamientos convencionales sobre diente no son posibles.

Citar como: Anitua E. Retratamiento de un caso con atrofia ósea compleja combinada unida a periimplantitis. Importancia de la reversibilidad de los tratamientos implantológicos. Rev Cient Odontol (Lima). 2020; 8(2): 023.

DOI: $10.21142 / 2523-2754-0802-2020-023$
Hoy en día, nos enfrentamos a la rehabilitación de casos cada vez más complejos, y aunque existen cada vez más y mejores técnicas para abordarlos no dejan de suponer un reto para el profesional $\left({ }^{1}\right)$. Esto se suma a la existencia de un número creciente de tratamientos previos de implantes «fracasados», principalmente por

1 Fundación Eduardo Anitua, BTI Biotechnology Institute. Vitoria, España. 
periimplantitis, aunque también se registran casos por fractura del implante o de los aditamentos protésicos, lo que inhabilita al implante para continuar sosteniendo una rehabilitación protésica ${ }^{(2-5)}$.

La unión de pacientes con atrofias severas que demandan tratamiento implantológico, junto con casos de severas atrofias en los que se debe realizar la sustitución de los implantes fallidos, complica aún más la consecución de un tratamiento de implantes con éxito. En muchos casos, se precisa la reconstrucción del lecho receptor antes de la colocación de los implantes, debido a que no existe un remanente óseo suficiente para la inserción directa de los implantes, por lo que el tratamiento resulta mucho más costoso económica y quirúrgicamente para el paciente. Para llevar a cabo esta reconstrucción, podemos emplear diferentes métodos, como injertos en bloque, regeneración ósea guiada, elevación del seno, distracción y técnicas de expansión entre otras $\left(^{6}\right)$. Al margen de la regeneración, se describen cada vez más técnicas que se englobarían dentro de la "cirugía mínimamente invasiva”, que pretende la rehabilitación del paciente con un número menor de técnicas quirúrgicas y comorbilidades asociadas. Entre estas técnicas se pueden describir los implantes cortos y extracortos, los implantes estrechos, la elevación del seno con abordaje crestal y las técnicas de crecimiento vertical alrededor de los implantes cortos y extracortos $\left(^{7-15}\right)$.

En esta misma línea de mínima intervención, se han desarrollado sistemas que permiten retirar los implantes fracasados y conservar al máximo el lecho óseo en el que asientan, para efectuar retratamientos con implantes dentales incluso en los casos más extremos $\left({ }^{16-18}\right)$. Estas técnicas nos permiten colocar nuevos implantes en casos extremos y utilizando muchas veces incluso el mismo lecho del implante retirado para la inserción de uno nuevo ${ }^{19-22}$. En algunos estudios consultados respecto de esta técnica de extracción e implantación de un nuevo implante,semencionan rangos de supervivencia diferentes del nuevo implante, que varían entre el 75 y el 88\% (19-23). Este hecho podría relacionarse con otro bien conocido en la literatura implantológica, que es la supervivencia de los implantes en pacientes afectados de periodontitis. En estos sujetos, la tasa de supervivencia de los implantes es inferior, situándose alrededor del $80 \%$, aun cuando esta se encuentra controlada, un $9 \%$ menos que los pacientes que no presentan este tipo de enfermedad, según los datos recopilados en la literatura internacional $\left({ }^{24-26}\right)$. En el siguiente caso clínico, mostramos a un paciente con un edentulismo de larga evolución tratado previamente con implantes inferiores y sobredentadura, pero que, debido al fracaso del tratamiento desde el punto de vista estético y funcional, acudió a la consulta para iniciar nuevo tratamiento implantológico. En él se recogen, por lo tanto, diferentes técnicas mínimamente invasivas para la rehabilitación como la explantación atraumática y la inserción de implantes cortos y extracortos, para evitar el abordaje del seno en el maxilar superior y la movilización del nervio dentario en la mandíbula.

\section{Caso clínico}

E1 caso reportado corresponde a una paciente de sexo femenino de 76 años que acudió a la consulta odontológica para rehabilitación sobre implantes. Presenta una prótesis removible superior desajustada con mucha movilidad en la masticación y una prótesis inferior sobre implantes removible que ha perdido fijación y produce dolor durante la masticación en los sectores posteriores, además de supuración espontánea. Las imágenes intraorales nos muestran ambas prótesis y nos hacen predecir la extrema reabsorción que presenta el hueso debido a la cresta ósea y la mucosa de extrema delgadez (figura 1).
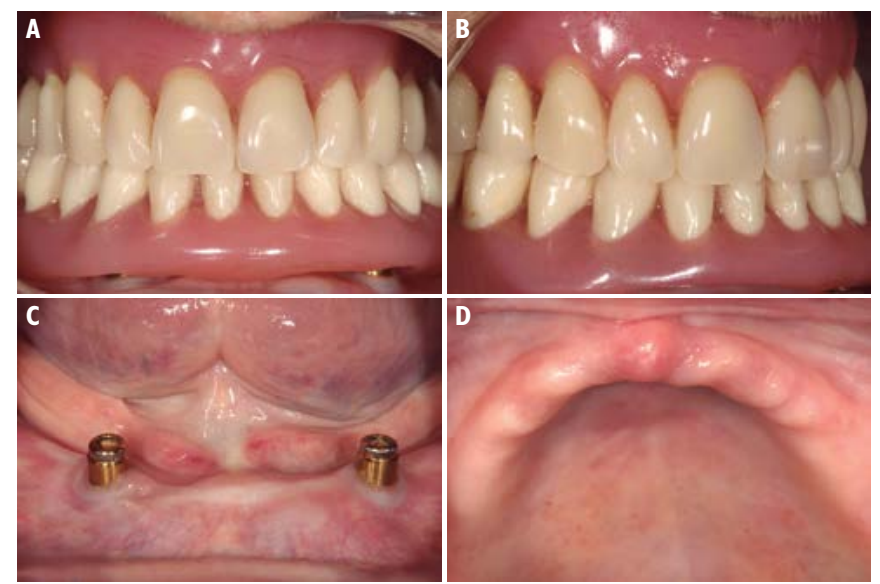

Figura I. Imágenes intraorales de la paciente a la llegada a la clínica con sus prótesis removibles convencionales $(a, b)$ y sin ellas (c, d). 
En la radiografía panorámica puede observarse, además, una pérdida ósea alrededor de ambos implantes, con sondaje de más de $5 \mathrm{~mm}$, sangrado y supuración al sondaje. Por lo tanto, se decidió la extracción de estos implantes y la inserción de unos nuevos en los arcos superior e inferior (figura 2).

En la mandíbula, se decidió la explantación de ambos implantes mediante el sistema de extracción atraumática ${ }^{(17-18)}$. En la planificación de los nuevos implantes mandibulares, se optó por la inserción en zonas adyacentes a los antiguos implantes sin utilizar los alveolos posexplantación. Esto se debe a motivos biomecánicos de reparto de los implantes, para aprovechar al máximo el volumen óseo residual ajustando los dos implantes más distales por lingual del foramen dentario y disponiendo los dos entre estos a distancia equidistante de la línea media (figura 3).

En el arco superior, la planificación contempló la inserción de implantes cortos y extracortos, debido a la existencia de una extrema reabsorción vertical que afectaba prácticamente todo el maxilar superior. Con la inserción de estos implantes, se evitaron técnicas más complejas de reconstrucción ósea que implicaran injertos en bloque, elevación del seno y otras técnicas de regeneración. De esta forma, se logró una menor cantidad de intervenciones quirúrgicas, a la vez que se evitaron posibles complicaciones derivadas de estas (figura 4).

Previamente a la cirugía, se premedicó al paciente con 2 gr de amoxicilina vía oral y paracetamol. Estos fármacos se administraron una hora antes de la intervención. Se

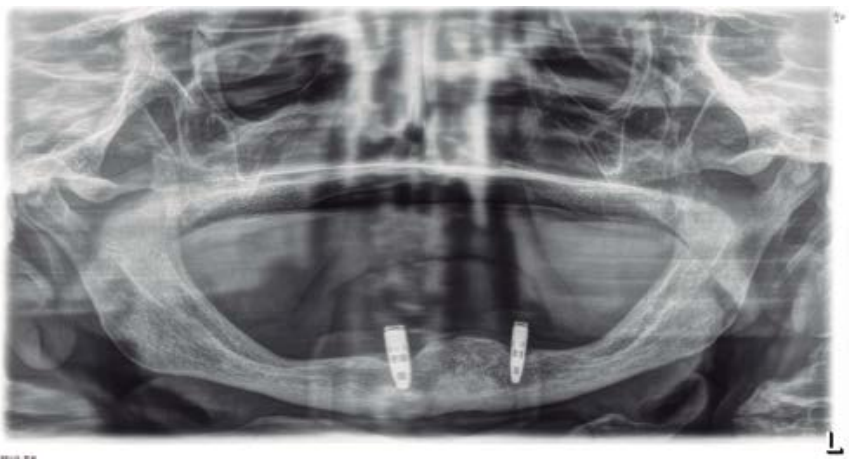

Figura 2. Radiografía inicial en la que se puede observar la posición desfavorable de los implantes remanentes, así como dos defectos circunferenciales alrededor de los mismos. llevó a cabo la cirugía superior e inferior en un mismo acto quirúrgico, y se logró una estabilidad suficiente en los implantes inferiores para la confección de una prótesis de carga inmediata, la cual fue colocada en las
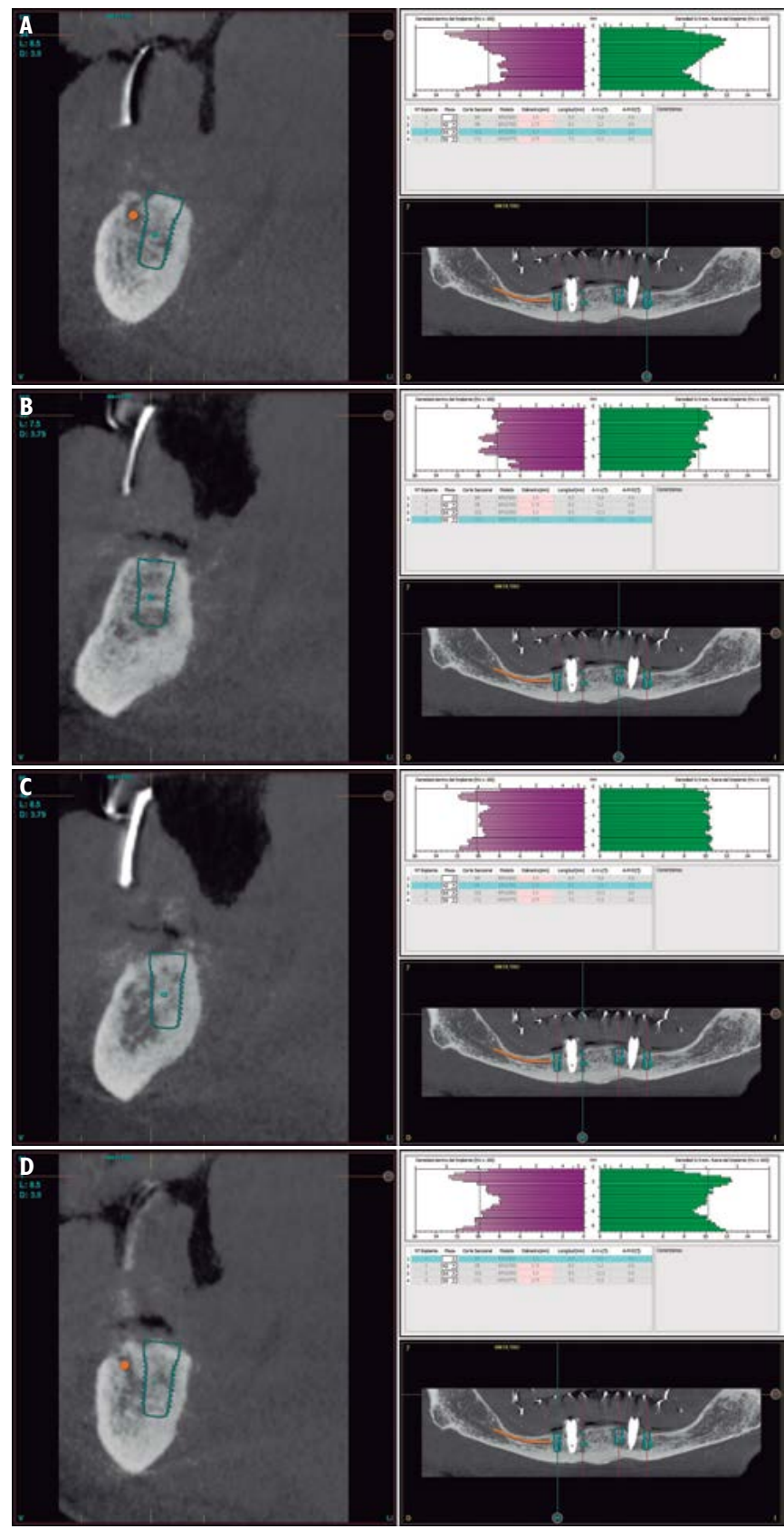

Figura 3. Cortes de planificación del TAC inferior en los que se puede observar la nueva distribución de los cuatro implantes que fueron insertados. Los dos más distales se colocaron ajustados a la salida del nervio dentario, incluso por distal de la misma para disminuir el voladizo distal el máximo posible. Los otros dos implantes se colocaron equidistantes a la línea media y a los nuevos implantes, por lo que las posiciones de los antiguos implantes no son correctas (a-d). 

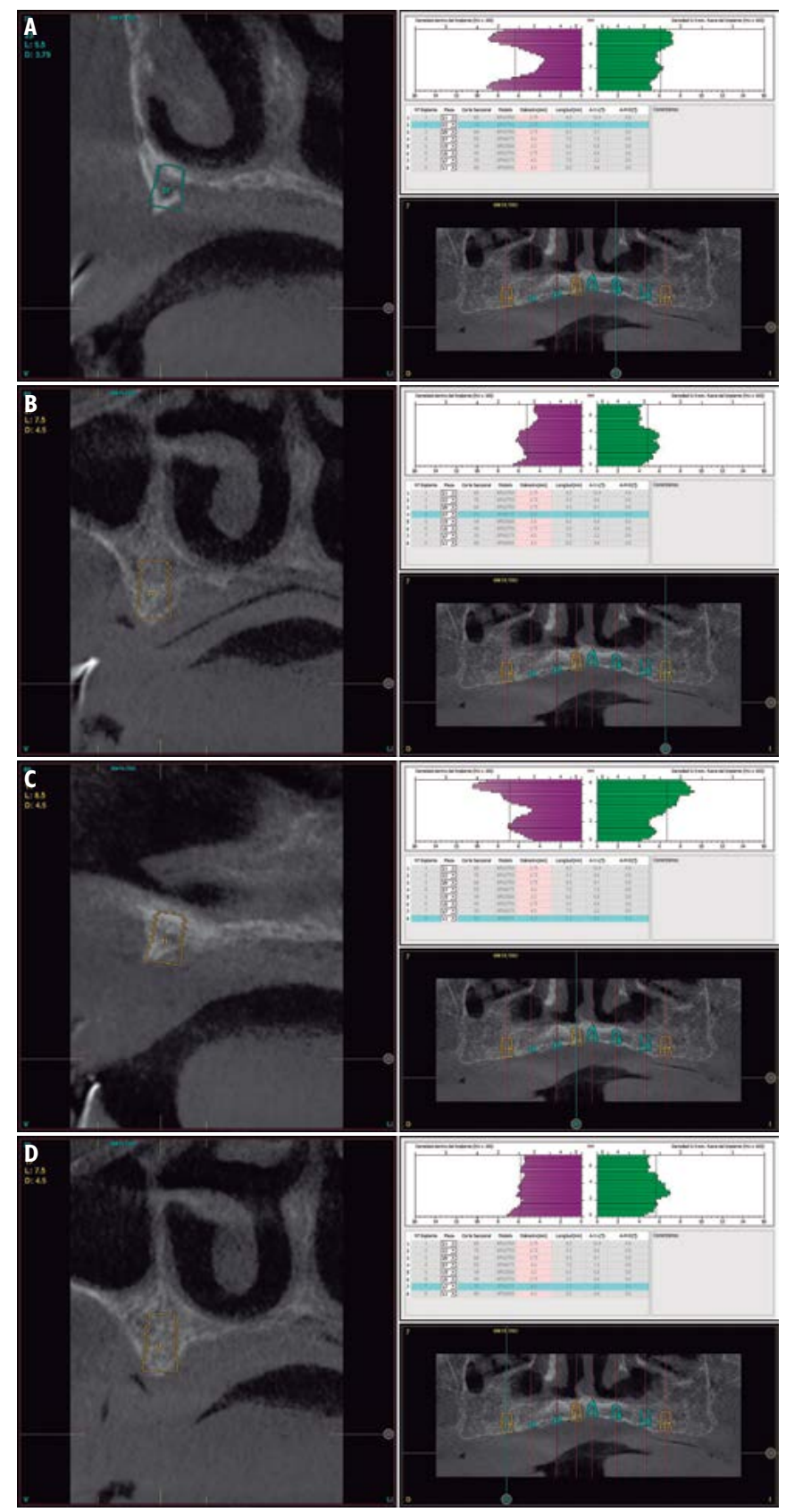

Figura 4. Imágenes de planificación del TAC superior en las cuales se puede observar la gran reabsorción horizontal que presenta el hueso maxilar en general, lo que obligó a la inserción de implantes cortos y extracortos (a-d).

primeras 24 horas gracias al uso de las barras articuladas para ensamblar la estructura de forma rápida y precisa. Los implantes inferiores fueron explantados a la vez que se insertaron los nuevos, y se pudo observarse cómo se preservó el lecho óseo sin perder nada del mismo por la extracción. En el arco superior, la estabilidad de los implantes quedó más comprometida, debido a la menor

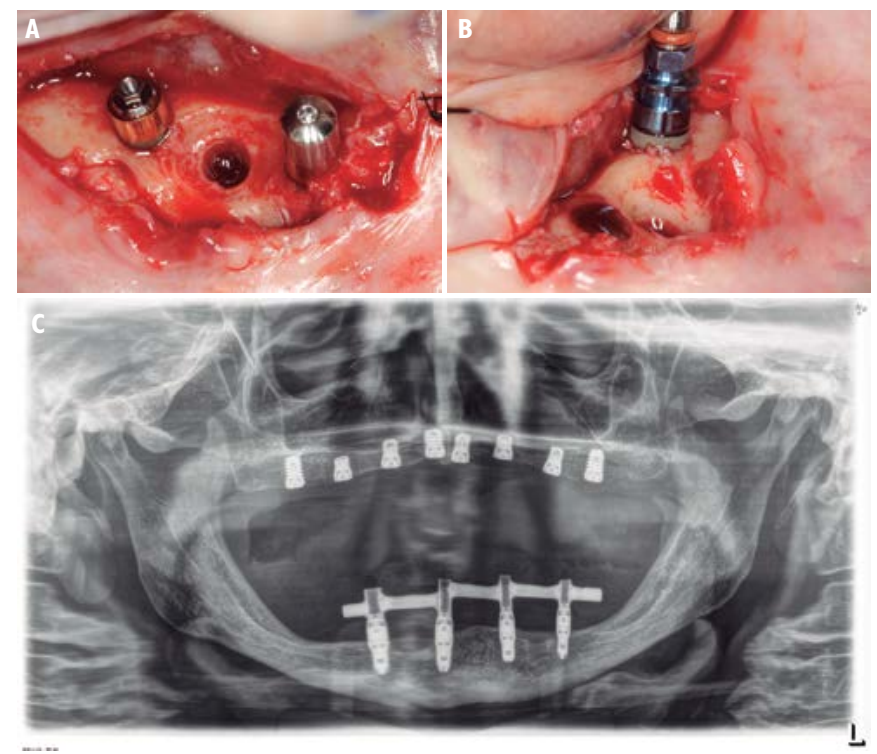

Figura 5. Cirugía de explantación junto con la inserción de los implantes inferiores. En el mismo acto quirúrgico se insertaron, además, los implantes superiores (a, b). La carga inmediata pudo ser llevada a cabo en la mandíbula por la densidad ósea. En el maxilar superior, al tener menor densidad, se realizó una carga diferida (dos fases). La prótesis inferior se confeccionó con las barras articuladas, por lo que pudo estar lista en 24 horas (c).

densidad del hueso receptor, por lo que se difirió la carga (figura 5).

Transcurridos seis meses desde la cirugía del arco superior, se confeccionó una prótesis de carga progresiva para esta zona. Del mismo modo que en el arco inferior, se utilizaron barras articuladas para generar la estructura de la prótesis superior. Con este tipo de prótesis se consigue una carga paulatina sobre los implantes con menor torque y en huesos de baja densidad. Una vez transcurrido un tiempo (2-3 meses), con estas prótesis podemos elaborar la definitiva, dándole tiempo también a la oclusión y la articulación temporomandibular para acomodarse a la nueva dimensión vertical (figura 6).

Luego de tres meses de la carga progresiva superior, se procedió a la confección de ambas prótesis definitivas (superior e inferior). Para ello, se realizó un encerado diagnóstico en el que se incorporaron los cambios que se deseaba introducir respecto de los provisionales (figura 7).

Una vez corregidos los ajustes estéticos (corrección de línea media de la prótesis con la línea media facial, 

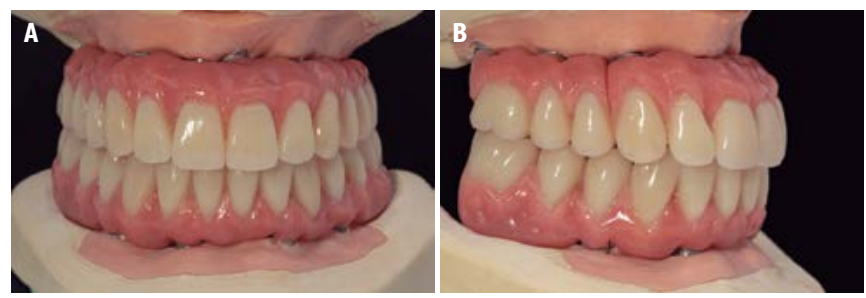

Figura 8. Confección de la estructura de la prótesis por CAD-CAM y terminación de la prótesis (a). La prótesis superior se realizó en tres tramos para facilitar la flexión del hueso en la masticación y eliminar tensiones (b).
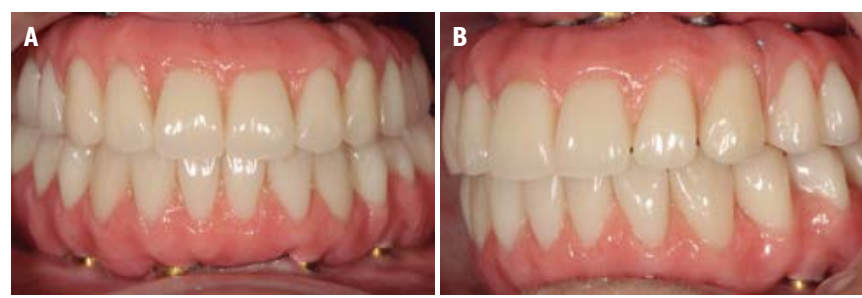

Figura 6. Tras 6 meses de la inserción de los implantes superiores, se procedió a la segunda fase y a la confección de una prótesis de carga progresiva superior (a, b). Esta prótesis, al igual que la de carga inmediata, se elaboró de forma rápida y sencilla con las barras articuladas (c).
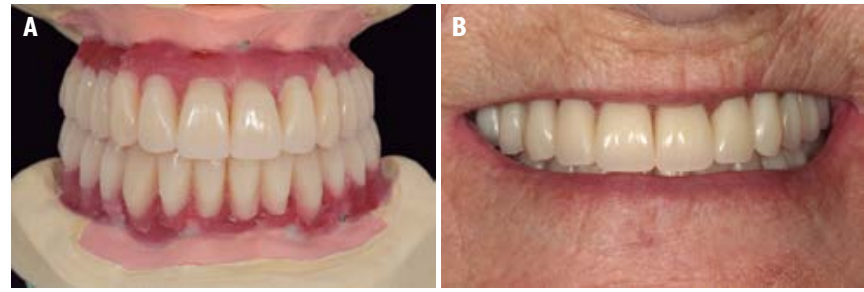

Figura 7. Encerado para la confección de la prótesis definitiva y prueba en boca para corregir los elementos estéticos y funcionales necesarios (a). En la fotografía se muestra una desviación de la línea media dental con respecto a la facial además de una exposición desigual de los incisivos centrales (b). Estos puntos se corrigieron para la confección de la prótesis definitiva.

exposición de diente en sonrisa y plano oclusal) se trasladó el encerado definitivo al laboratorio. La estructura que se construyó por CAD-CAM para corregir las angulaciones de las emergencias de los tornillos y posicionarlas en zonas donde no produjeran problemas estéticos. Además, se fabricó la prótesis superior en tres tramos, debido a que se contaba con implantes suficientes para ello, y esto permitió la flexión del hueso de manera natural en la masticación y un mejor ajuste pasivo (figura 8).

Una vez terminadas, ambas prótesis se colocaron en la paciente. Se puede observar en las fotografías cómo se ha logrado recuperar la estética y, por supuesto, una mejor función que con las prótesis iniciales. El aumento

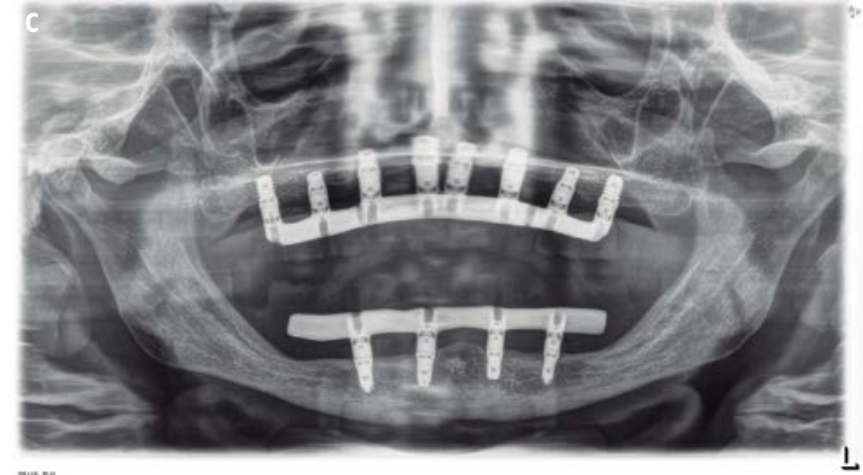

Figura 9. Prótesis colocada en la paciente en visión frontal (a) y lateral (b) Radiografía con la prótesis terminada al año de la colocación (c). Se puede observar cómo los implantes se encuentran estables sin pérdidas óseas.

de la dimensión vertical que estaba colapsada permitió, además, recuperar una mejor función de la articulación temporomandibular (figura 9).

En las siguientes imágenes se puede apreciar la comparación del antes y el después del tratamiento, tanto en las prótesis como en la radiografía (figura 10). Posteriormente, se programaron visitas de seguimiento al mes, tres meses, seis meses y, finalmente, una vez al año, sin que se presentaran incidencias en ninguna de ellas.

\section{Discusión}

Las atrofias mandibulares y maxilares severas son una consulta habitual hoy en día en la clínica dental, por 

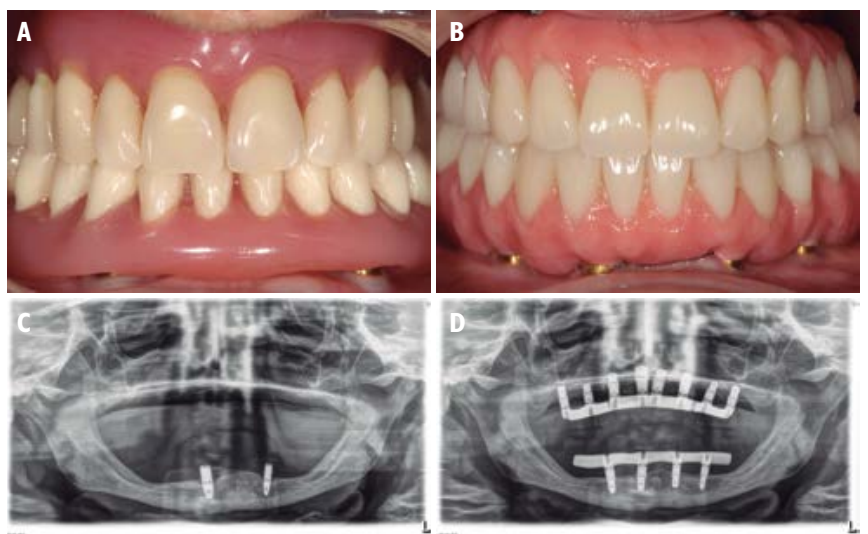

Figura 10. Comparativa del estado inicial (prótesis y radiografía) (a, c) con el estado final $(b, d)$, después de tres años de seguimiento.

lo que el profesional debe estar familiarizado con las diferentes técnicas de tratamiento, sobre todo las que implican un menor número de cirugías y disminuyen la morbilidad del paciente, ya que estos procedimientos son mejor aceptados $\left({ }^{27-32}\right)$. Los implantes cortos y extracortos son una opción segura para la rehabilitación de sectores posteriores maxilares y mandibulares, como alternativa a técnicas de aumento óseo más complejas, con cifras de supervivencia a largo plazo superiores al 98\%, una menor comorbilidad asociada y una simplificación de las técnicas quirúrgicas $\left.{ }^{8-12}\right)$.

Realizar la extracción y la nueva inserción de los implantes fracasados o implantes comprometidos por periimplantitis en un mismo acto quirúrgico es también una ventaja para el retratamiento de los casos de implantes fracasados, sobre todo en casos extremos como el que mostramos. Cuando unimos, además, la carga inmediata, como en el caso de la mandíbula, nos permite rehabilitar al paciente con una prótesis mucho más funcional y de forma más rápida, sin disminuir la predictibilidad del tratamiento. Estudios de carga inmediata en implantes cortos y extracortos muestran supervivencias acumuladas entre el $87 \%$ y el $97 \%\left({ }^{33-37}\right)$, por lo que no aumentan en exceso los fracasos comparados con los implantes cortos y extracortos con carga convencional.

Para lograr éxito en los abordajes mínimamente invasivos, como los implantes cortos y extracortos con carga inmediata, intervienen una serie de puntos clave que tienen una importancia posterior en las tasas de éxito. Estos factores pueden agruparse principalmente en tres tipos: dependientes del hueso remanente, dependientes del acto quirúrgico y dependientes del tipo de prótesis implanto-soportada que se ha seleccionado ${ }^{(38)}$. Uno de los principales factores es la densidad ósea, que determinará parte del torque de inserción final que se logrará en la cirugía y, por lo tanto, es un factor importante que habilitará la carga inmediata $\left({ }^{39}\right)$. A una mayor densidad del lecho receptor del implante, es probable que se obtenga una mayor estabilidad en la inserción, si se ha seguido un cuidadoso protocolo de fresado. Por eso, en el maxilar superior se optó por una carga diferida para evitar una sobrecarga funcional.

\section{Conclusiones}

En casos de atrofia severa maxilar y mandibular, el uso de los implantes cortos puede acortar los tiempos de tratamiento y simplificar las técnicas quirúrgicas, sin renunciar a la predictibilidad, como en el caso mostrado.

Contribución de los autores: Un único contribuyente ha realizado el artículo: Eduardo Anitua.

Conflicto de intereses: Eduardo Anitua es el director científico de BTI.

Consideraciones éticas: El paciente incluido en el estudio firmó un consentimiento informado. 


\section{REFERENCIAS BIBLIOGRÁFICAS}

1. Buser D, Sennerby L, De Bruyn H. Modern implant dentistry based on osseointegration: 50 years of progress, current trends and open questions. Periodontol 2000. 2017; 73: 7-21. doi: 10.1111/prd.12185

2. Solderer A, Al-Jazrawi A, Sahrmann P, Jung R, Attin T, Schmidlin PR. Removal of failed dental implants revisited: Questions and answers. Clin Exp Dent Res. 2019; 5: 712-24. doi: $10.1002 /$ cre2.234

3. Caplanis N, Kusek E, Low S, Linden E, Sporborg H. Periimplant disease, a consensus for treatment: a case study. J Oral Implantol. 2019; 45: 371-7. doi: 10.1563/aaid-joi-D-19-00043

4. Robertson K, Shahbazian T, MacLeod S. Treatment of periimplantitis and the failing implant. Dent Clin North Am. 2015; 59: 329-43. doi: 10.1016/j.cden.2014.10.007

5. Greenstein G, Cavallaro J. Failed dental implants: diagnosis, removal and survival of reimplantations. J Am Dent Assoc. 2014; 145: 835-42. doi: 10.14219/jada.2014.28

6. Baj A, Trapella G, Lauritano D, Candotto V, Mancini GE, Gianni AB. An overview on bone reconstruction of atrophic maxilla: success parameters and critical issues. J Biol Regul Homeost Agents. 2016; 30: 209-15.

7. Misch CM, Polido WD. A "graft less" approach for dental implant placement in posterior edentulous sites.Int J Periodontics Restorative Dent. 2019; 39: 771-9. doi: 10.11607/prd.4414

8. Zhou X, Hu XL, Li JH, Lin Y. Minimally invasive crestal sinus lift technique and simultaneous implant placement. Chin J Dent Res. 2017; 20: 211-8. doi: 10.3290/j.cjdr.a39220

9. Mijiritsky E, Barbu H, Lorean A, Shohat I, Danza M, Levin L. Use of implant-derived minimally invasive sinus floor elevation: a multicenter clinical observational study with $12-$ to 65 -month follow-up. J Oral Implantol. 2016; 42: 343-8. doi: 10.1563/aaidjoi-D-15-00157

10. Hof M, Tepper G, Semo B, Arnhart C, Watzek G, Pommer B. Patients perspectives on dental implant and bone graft surgery: questionnaire-based interview survey. Clin Oral Implants Res. 2014; 25: 42-5. doi: 10.1111/clr.12061

11. Schwartz SR. Short implants: an answer to a challenging dilemma? Dent Clin North Am. 2020; 64: 279-90. doi: 10.1016/j. cden.2019.11.001

12. Lozano-Carrascal N, Anglada-Bosqued A, Salomó-Coll O, Hernández-Alfaro F, Wang HL, Gargallo-Albiol J. Short implants $(<8 \mathrm{~mm})$ versus longer implants $(\geq 8 \mathrm{~mm})$ with lateral sinus floor augmentation in posterior atrophic maxilla: A metaanalysis of RCT's in humans. Med Oral Patol Oral Cir Bucal. 2020; 25: e168-e179. doi: 10.4317/medoral.23248

13. Vazouras K, de Souza AB, Gholami H, Papaspyridakos P, Pagni $\mathrm{S}$, Weber HP. Effect of time in function on the predictability of short dental implants ( $\leq 6 \mathrm{~mm})$ : A meta-analysis. J Oral Rehabil. 2020; 47: 403-15. doi: 10.1111/joor.12925

14. Telles LH, Portella FF, Rivaldo EG. Longevity and marginal bone loss of narrow-diameter implants supporting single crowns: A systematic review. PLoS One. 2019; 14: e0225046. doi: 10.1371/journal.pone.0225046
15. Ma M, Qi M, Zhang D, Liu H. The clinical performance of narrow diameter implants versus regular diameter implants: a meta-analysis. J Oral Implantol. 2019; 45: 503-8. doi: 10.1563/ aaid-joi-D-19-00025

16. Stajčić Z, Stojčev Stajčić LJ, Kalanović M, Đinić A, Divekar N, Rodić M. Removal of dental implants: review of five different techniques. Int J Oral Maxillofac Surg. 2016; 45: 641-8. doi: 10.1016/j.ijom.2015.11.003

17. Anitua E, Murias-Freijo A, Piñas L, Tejero R, Prado R, Orive G. Nontraumatic implant explantation: a biomechanical and biological analysis in sheep tibia. J Oral Implantol. 2016; 42: 3-11. doi: 10.1563/aaid-joi-D-14-00193

18. Anitua E, Murias-Freijo A, Alkhraisat MH. Conservative implant removal for the analysis of the cause, removal torque, and surface treatment of failed nonmobile dental implants. J Oral Implantol. 2016; 42: 69-77. doi: 10.1563/aaid-joi-D-14-00207

19. Anitua E, Orive G. A new approach for atraumatic implant explantation and immediate implant installation. Oral Surg Oral Med Oral Pathol Oral Radiol. 2012; 113: e19-25. doi: 10.1016/j. tripleo.2011.06.035

20. Covani U, Barone A, Cornelini R, Crespi R. Clinical outcome of implants placed immediately after implant removal.J Periodontol. 2006; 77: 722-7.

21. Covani U, Marconcini S, Crespi R, Barone A. Immediate implant placement after removal of a failed implant: a clinical and histological case report. J Oral Implantol. 2009; 35: 189-95. doi: 10.1563/1548-1336-35.4.189

22. Covani U, Marconcini S, Santini S, Cornelini R, Barone A. Immediate restoration of single implants placed immediately after implant removal. A casereport. Int J Periodontics Restorative Dent. 2010; 30: 639-45.

23. Grossmann Y, Levin L. Sucess and survival of single dental implants placed in sites of previously failed implants.J Periodontol 2007; 78: 1670-4.

24. Heitz-Mayfield LJ,Huynh-Ba G. History of treated periodontitis and smoking as risks for implant therapy. Int J Oral Maxillofac Implants. 2009; 24 Suppl:39-68.

25. Greenstein G, Cavallaro J Jr, Tarnow D. Dental implants in the periodontal patient. Dent Clin North Am. 2010; 54: 113-28. doi: 10.1016/j.cden.2009.08.008

26. Safii SH, Palmer RM, Wilson RF. Risk of implant failure and marginal bone loss in subjects with a history of periodontitis: a systematic review and meta-analysis. Clin Implant Dent Relat Res. 2010; 12: 165-74. doi: 10.1111/j.1708-8208.2009.00162.x

27. Edelmayer M, Woletz K, Ulm C, Zechner W, Tepper G. Patient information on treatment alternatives for missing single teeth Systematic review. Eur J Oral Implantol. 2016; 9 Suppl 1: S4557.

28. Gurgel BC, Pascoal AL, Souza BL, Dantas PM, Montenegro SC, Oliveira AG, Dos Santos P. Patient satisfaction concerning implant-supported prostheses: an observational study. Braz Oral Res. 2015; 29. doi: 10.1590/1807-3107BOR-2015.vol29.0034 
29. Pommer B, Mailath-Pokorny G, Haas R, Busenlechner D, Fürhauser R, Watzek G. Patients' preferences towards minimally invasive treatment alternatives for implant rehabilitation of edentulous jaws. Eur J Oral Implantol. 2014;7 Suppl 2: S91-109.

30. Esposito M, Cannizzaro G, Soardi E, Pistilli R, Piattelli M, Corvino V, Felice P. Posterior atrophic jaws rehabilitated with prostheses supported by $6 \mathrm{~mm}$-long, $4 \mathrm{~mm}$-wide implants or by longer implants in augmented bone. Preliminary results from a pilot randomised controlled trial. Eur J Oral Implantol. 2012; 5: 19-33.

31. Checchi L, Felice P, Antonini ES, Cosci F, Pellegrino G, Esposito M. Crestal sinus lift for implant rehabilitation: a randomised clinical trial comparing the Cosci and the Summers techniques. A preliminary report on complications and patient preference. Eur J Oral Implantol. 2010; 3: 221-32.

32. Felice P, Checchi V, Pistilli R, Scarano A, Pellegrino G, Esposito $\mathrm{M}$. Bone augmentation versus $5-\mathrm{mm}$ dental implants in posterior atrophic jaws. Four-month post-loading results from a randomised controlled clinical trial. Eur J Oral Implantol. 2009; 2: 267-81.

33. Cannizzaro G, Leone M, Torchio C, Viola P, Esposito M. Immediate versus early loading of 7 -mm-long flapless-placed single implants: a split-mouth randomised controlled clinical trial. Eur J Oral Implantol. 2008; 1: 277-92.
34. Degidi M, Piattelli A, Iezzi G, Carinci F. Immediately loaded short implants: analysis of a case series of 133 implants. Quintessence Int. 2007; 38: 193-201.

35. Anitua E, Flores C, Flores J, Alkhraisat MH. Clinical Effectiveness of 6.5-mm-long implants to support two-implant fixed prostheses in premolar-molar region: the influence of immediate loading and the length of splinting implant. J Prosthodont. 2019; 28: e688-e693. doi: 10.1111/jopr.12761

36. Alvira-González J, Díaz-Campos E, Sánchez-Garcés MA, GayEscoda C. Survival of immediately versus delayed loaded short implants: A prospective case series study. Med Oral Patol Oral Cir Bucal. 2015; 20: e480-8.

37. Maló P, de Araújo Nobre MA, Lopes AV, Rodrigues R. Immediate loading short implants inserted on low bone quantity for the rehabilitation of the edentulous maxilla using an Allon-4 design. J Oral Rehabil. 2015; 42: 615-23. doi: 10.1111/ joor.12291

38. Gapski R, Wang HL, Mascarenhas P, Lang NP. Critical review of immediate implant loading. Clin Oral Implants Res 2003; 14 : 515-27.

39. Barewal RM, Stanford C, Weesner TC. A randomized controlled clinical trial comparing the effects of three loading protocols on dental implant stability. Int J Oral Maxillofac Implants 2012; 27: 945-56. 